\title{
Understanding the local structure of disordered carbons from cellulose and lignin
}

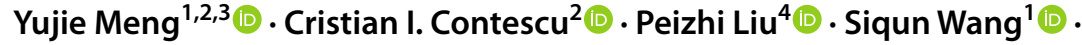 \\ Seung-Hwan Lee ${ }^{5}$ (D) Junjie Guo ${ }^{2,4} \cdot$ Timothy M. Young $^{1}{ }^{1(1)}$
}

Received: 1 July 2020 / Accepted: 23 March 2021 / Published online: 15 April 2021

(c) The Author(s) 2021

\begin{abstract}
An electron microscopy investigation was performed to understand the relationship between the microstructure and properties of carbonized cellulose and lignin (softwood kraft lignin) relative to the structure of the original biomass components. Structure details at micro- and molecular levels were investigated by scanning transmission electron microscopy. Atomic-resolution images revealed the presence of random disordered carbon in carbonized cellulose (C-CNC) and of large domains of well-ordered carbon with graphite sheet structure in carbonized lignin (C-Lignin). These structural differences explain why $\mathrm{C}-\mathrm{CNC}$ exhibits higher surface area and porosity than C-Lignin. The presence of certain well-ordered carbon in carbonized lignin indicates some of the carbon in lignin are graphitized with heat treatment temperature up to $950{ }^{\circ} \mathrm{C}$. This result is encouraging for future endeavors of attaining acceptable modulus of carbon fiber from lignin given suitable modifications to the chemistry and structure of lignin. The results of this research contribute to an improved understanding of the carbonization mechanism of the key cellulose and lignin components of biomass materials.
\end{abstract}

\section{Introduction}

By converting biomass into fuels, power, materials and chemicals, biorefining is gradually making contributions to sustainable industrial development (Ragauskas et al. 2006; Aarum et al. 2019). Biomass is a combination of three major biopolymers. First, cellulose is an aggregate of linear chains of glucopyranose units linked by $\beta$ - $(1,4)$ glycosidic bonds to varying degrees of polymerization. The chains held together in parallel stacks by intra- and intermolecular hydrogen bonds form shortrange nanocrystalline regions. The cellulose nanocrystals (CNCs) alternate with amorphous regions and form microfibrils held by weak van der Waals forces (Moon

Timothy M. Young

tmyoung1@utk.edu

Extended author information available on the last page of the article 
et al. 2011; Wu et al. 2013; Trache et al. 2017; Risnasari et al. 2018; Wang et al. 2020). Second, hemicellulose consists of wide groups of highly branched heteroglycans. Third, lignin is a complex polymer of aromatic alcohols (monolignols) composed mainly of three phenylpropane monomers: $p$-coumaryl alcohol, coniferyl alcohol, and sinapyl alcohol.

Bioresources are abundant, renewable, sustainable, biocompatible and biodegradable; which have the most abundant natural aromatic polymers in the world (Langan et al. 2014). Converting biomass into carbon materials is of tremendous interest in the development of high-value added products used for energy storage and conversion in batteries and supercapacitors, environment remediation, gas separation and storage, oil absorption, drug delivery, catalyst, etc. (Chen et al. 2019; Meng et al. 2014; He et al. 2014; Dobele et al. 2012; Wang et al. 2013; Zhao et al. 2012). The increased interest in biomass conversion into usable products is exemplified by a series of recent studies. These studies include synthesizing graphene from rice husk and hemp (Wang et al. 2013; Muramatsu et al. 2014), investigating onion-like graphitic and diamond structures in wood charcoal (Hata et al. 2000; Ishimaru et al. 2007; Goodell et al. 2008), constructing three-dimensional porous carbon from waste diapers (Wei et al. 2018), and obtaining carbon nanotubes from carbonization of grass and wood hierarchically structured fibers (Goodell et al. 2008). In essence, synthesis of carbonaceous materials occurs through a complex process involving oxidation, pyrolysis, and carbonization.

The promise of manufacturing low-cost, high-performance carbon fibers from lignin and cellulose precursors, a main driver of the bio refining concept, still faces great challenges and is not yet ready for industrial-scale production and application. Current applications of lignin carbon fiber with the new concepts of non-structural, multifunctional include active electrodes in batteries, hydrogen gas storage material, thermal insulation material, and separators in super capacitors (Mao et al. 2015). Carbon nanofiber generated from cellulose nanofibers has been used as a reinforcement in thermoplastics to improve the resulted composite's tensile strength (Ma et al. 2016).

One problem to be solved is the poor mechanical properties of resulting products with porous and disordered structures. Moreover, there exists a certain link between the carbon precursor chemistry and the final morphology of carbonized material. Attempts have been made to study and compare the oxidative stabilization and carbonization kinetics of cellulose and lignin using only spectroscopic analysis. Results indicate that lignin and cellulose exhibit different carbon to oxygen ratios, which drastically affects the conversion mechanism, which influences the microstructure of the resulting carbon (Ishimaru et al. 2007; Cao et al. 2013; Xie et al. 2009). Nonetheless, understanding the structure-property relationship of biomass-derived carbonaceous materials at the microscale remains a significant research challenge. Attempts have been made to observe the biochar's molecular structure at atomic level. Xiao and Chen (2017) proposed a quaternary structure for biochars, which includes heterogeneous phase, graphene-like aggregates, nanosized aromatic clusters and atomic arrangements. However, it is still not clear how does each component play its role during the biomass conversion process and how do they interact with each other. As such, the object of this research was to investigate the structure 
evolution of each component in biomass in the thermo decomposition process. Typically, nanocellulose and lignin were used as the representative precursors as they manifest very different structures.

Recently, the investigation of the local structure of porous carbon has been made possible by the development of atomic-resolution electron microscopy. From this technology, it has been recently demonstrated that the nanoscale structure of woodbased carbons contains randomly stacked, wrinkled graphene sheets (He et al. 2014; Guo et al. 2012). Their variable architectural arrangement at the microscale creates a network of interconnected nanopores with large internal surfaces, which determine the main functional property of porous carbons. At the atomic scale, the curvature of individual graphene/graphite sheet structure is determined by topological defects, such as 5- and 7-atom carbon rings, randomly interspersed between regular 6-atom carbon rings. With this complex architecture, porous carbons exhibit shortrange atomic order at the sub-nanometer scale, but appear as an isotropic, amorphous material at the microscale. The complicated structure and varying chemical composition of wood components represent a significant challenge for improving the understanding of the carbonization mechanism of each of the components. The origin of locally ordered graphite sheet structures and the formation mechanism of biomass-based porous carbon are still somewhat elusive at the atomic level. The research hypothesis of this study was that variability in biomass precursor's components increases the structural diversity of the resultant carbon materials.

Direct imaging of CNC by means of the high-resolution transmission microscope (HRTEM) is important to probe and manipulate the size-dependent physical and chemical properties of CNC at the nanoscale (Aina et al. 2013). Very few researchers to date have captured the lattice structure of CNC due to the difficulty in sample preparation and contamination issue in TEM technology. CNC has a low electronscattering power when under TEM, as such a negative-staining technique has been used to prepare the CNC sample for electron microscopy observation to enhance the contrast (Xu et al. 2013; Liu et al. 2011). However, these radioactive salts impede accurate observations. This research investigation successfully resolved the lattice structure of CNC without using negative staining by using a gentle scanning transmission electron microcopy (STEM) at a low accelerating voltage of $60 \mathrm{kV}$. This development is important for the fundamental understanding of structural transformations during cellulose conversion to carbon. The loss of crystallinity of the CNC structure caused from beam irradiation was also observed during this research.

In this work, two types of carbons obtained through identical carbonization conditions from cellulose nanocrystals and lignin were observed. It was found that under identical conditions, nanocrystals extracted from cellulose convert into disordered graphene-based nanoporous structures typical for porous carbons, while carbonized lignin forms non-porous mixtures containing well-ordered carbon with a graphite sheet structure, and globular onion-like inclusions dispersed throughout an amorphous matrix. The local atomic structure of the original CNC and of carbonized product, as well as lignin, was investigated for the first time. The local structural difference between the carbonized cellulose (C-CNC) and carbonized lignin (C-Lignin) explained the observed difference of porosity and surface area measured by the nitrogen adsorption method. This research is a noteworthy contribution to the 
improved understanding of the carbonization mechanism of the numerous biomass components.

\section{Materials and methods}

\section{Materials}

The CNC material studied here was derived from high-purity CF11 cellulose powder (Waterman, USA). Sulfuric acid $\left(\mathrm{H}_{2} \mathrm{SO}_{4}, 95-98 \%\right.$, Sigma-Aldrich) was used for acid hydrolysis. Indulin AT softwood kraft lignin sample was commercially available from MeadWestvaco (Charleston, SC).

\section{Preparation of cellulose nanocrystal and lignin}

CF11 cellulose was hydrolyzed by adding ten grams of powder to $100 \mathrm{~mL}$ sulfuric acid $(60 \mathrm{wt} \%)$ at $45{ }^{\circ} \mathrm{C}$ for 45 min under constant stirring. Cold distilled water was added to end the acid hydrolysis reaction. The hydrolyzed cellulose was washed, and nanocrystals were separated from the solution by centrifugation (4000 rpm, $20 \mathrm{~min}$ ). The precipitate was then dialyzed against water for four days using membrane with a molecular weight cutoff of 12,000-14,000 (Fisher brand, Pittsburgh, PA) until a constant neutral $\mathrm{pH}$ was achieved. The commercial lignin powder was washed with DI water to remove salt impurities, dried in vacuum at $80{ }^{\circ} \mathrm{C}$, and stored in the desiccant cabinet ready for use.

\section{Carbonization of cellulose nanocrystal and lignin}

CNC suspension (0.7 wt\%) was frozen using liquid nitrogen and was freeze-dried in a vacuum lyophilizer (Labconco Inc., Kansas City, MO) at a temperature of $-51{ }^{\circ} \mathrm{C}$ for three days. The dried CNC and lignin powder were stabilized in air $(20 \mathrm{~mL} / \mathrm{min})$ and then carbonized in nitrogen $(20 \mathrm{~mL} / \mathrm{min})$ using a Lindberg/blue M Mini-Mite tube furnace (Thermo Scientific). The process includes: (1) heating the sample from room temperature to $180{ }^{\circ} \mathrm{C}$ at a rate of $10^{\circ} \mathrm{C} / \mathrm{min}$ in air; (2) increasing the temperature from 180 to $230{ }^{\circ} \mathrm{C}$ at a rate of $5{ }^{\circ} \mathrm{C} / \mathrm{min}$ in air; (3) increasing the temperature from 230 to $320{ }^{\circ} \mathrm{C}$ at a rate of $2{ }^{\circ} \mathrm{C} / \mathrm{min}$ in air; and (4) increasing the temperature from 320 to $950{ }^{\circ} \mathrm{C}$ at a rate of $5{ }^{\circ} \mathrm{C} / \mathrm{min}$ in nitrogen and holding for $15 \mathrm{~min}$. The resulting samples were ground and dispersed into DI water. The mixture was sonicated continuously for 20 min with an ultrasonic probe.

\section{Nitrogen adsorption}

Nitrogen adsorption/desorption isotherms at $-196{ }^{\circ} \mathrm{C}$ were measured on a Quantachrome Autosorb $1 \mathrm{C}$ instrument with ASIQ Win 2.0 data processing software. Prior to measurements, samples were degassed for $1.5 \mathrm{~h}$ at $300{ }^{\circ} \mathrm{C}$ in vacuum of $(0.1 \mathrm{~Pa})$ to remove moisture and other volatile species. The pore surface area, pore volume, 
and pore size distribution were determined by the quenched surface density functional theory (QSDFT) model for slit/cylindrical pore geometry. In this case, open pores in the range of supermicropores $(0.7-2 \mathrm{~nm})$ and mesopores $(2-50 \mathrm{~nm})$ were investigated. Specific surface areas $\left(\mathrm{m}^{2} / \mathrm{g}\right)$ evaluated by Brunauer-Emmett-Teller method using nitrogen adsorption data at $-196{ }^{\circ} \mathrm{C}$ in the relative pressure $(\mathrm{p} / \mathrm{p} 0)$ range from 0.05 to 0.35 were also reported.

\section{Electron microscopy}

Suspension drops $(0.001 \mathrm{wt} \%)$ of CF11 cellulose nanocrystals were deposited on a holey silicon nitride support film (Ted Pella, Inc., USA) for TEM observation. The carbonized $\mathrm{CNC}$ and lignin samples were dispersed into deionized distilled water and treated with high-intensity ultrasonic processor (HIUS, $1500 \mathrm{~W}$ Model, SONICS Newtown, CT) to obtain the small fragments. A spin coating technique was used during the deposition to avoid aggregation of $\mathrm{CNC}$. The grids were placed into a high-resolution transmission electron microscope (Libra $200 \mathrm{MC}$, Zeiss), which was operated at a $200 \mathrm{kV}$ accelerating voltage. The atomic structure of CNC was studied using a 5th order spherical aberration corrected scanning transmission electron microscope (Nion-UltraSTEM100), which is operated at a low accelerating voltage of $60 \mathrm{kV}$ to avoid the irradiation damage to samples.

\section{Thermal gravimetric analysis}

The thermal stability and carbonization yield were characterized using thermal gravimetric analyzers (TGA; PerkinElmer 7 series; PerkinElmer Cetus Instruments, Norwalk, CT). The heating program was set up to mimic the carbonization process.

\section{Wide-angle X-ray diffraction}

X-ray diffraction measurements were carried out on a Scintag DMC 008 X-ray diffractometer with $\mathrm{Cu}$-target radiation at $40 \mathrm{kV}$ and $40 \mathrm{~mA}$ and a measuring angle from $5^{\circ}$ to $40^{\circ}$ (step size $=0.02^{\circ}$, scanning rate two sec/step). Powder samples were pressed into flat disks and attached to the glass plate. The crystallinity index (CrI) was calculated from diffraction patterns by using Eq. (1):

$$
\mathrm{CrI}=\left(\frac{I_{200}-I_{a m}}{I_{200}}\right) \times 100
$$

where $I_{200}$ is the intensity of $(200)$ peak $\left(2 \theta=22.4^{\circ}\right)$ and $I_{a m}$ is the minimum intensity at the valley between peaks (200) and (110) $\left(2 \theta=18^{\circ}\right)$. The lateral crystal dimension of the crystal with $h k l$ Miller indices, $D_{h k l}$, was calculated by Scherrer's expression (Patterson 1939):

$$
D_{h k l}=\frac{K \lambda}{\left(\beta_{\frac{1}{2}} \times \cos \theta\right)}
$$


where $K=0.94, \theta$ is the diffraction angle, $\lambda=0.154$ is the $\mathrm{X}$-ray wavelength and $\beta_{1 / 2}$ is the peak width at half maximum intensity of the $(h k l)$ peak.

\section{Fourier transform infrared spectroscopy (FTIR)}

FTIR was performed using a PerkinElmer FTIR-ATR spectrometer (Spectrum One, PerkinElmer, USA). Samples were placed on a diamond crystal of an attenuated total reflectance (ATR) accessory. All spectra were collected over a range of $4000-600 \mathrm{~cm}^{-1}$ at $4 \mathrm{~cm}^{-1}$ resolutions with 16 scans. Samples were recorded before and after carbonization.

\section{Raman spectrum analysis}

Carbon samples were examined by a micro-Raman spectrometer (Renishaw, UK) with an $\mathrm{Ar}+$ laser source (excitation wavelength $514.5 \mathrm{~nm}$ ). Calibration was done using the Raman active vibration peak at $520 \mathrm{~cm}^{-1}$ of silicon. All spectra were obtained with the laser power set to $100 \%$ with extended scans between 500 and $3000 \mathrm{~cm}^{-1}$ and were constructed using five separate measurements of $10 \mathrm{~s}$ each. Raman spectrum of carbonized CNC and carbonized lignin was deconvoluted into four peaks using Gauss and Lorentz function, where four peaks are centered at $\sim 1170, \sim 1345$ (D1 band), 1500 (D3 band) and 1600 (G band), respectively. The concentration of graphene disorder is extracted from the intensity ratio $I_{D} / I_{G}$ (Childres et al. 2013).

\section{Results and discussion}

\section{Porosity information from nitrogen as adsorption}

The results of porosity studies are plotted in Fig. 1. The N2 adsorption-desorption isotherms of C-CNC and C-Lignin feature typical type-II curves combined with H4-type hysteresis loop (Sing et al. 1985). This type of isotherms and the narrow hysteresis loops are attributed to interparticle capillary condensation.

For C-CNC, the adsorption amount undergoes a steep increase at relative pressure $(p / p 0)$ of $\sim 0.1$, indicating the completion of the first monolayer and transition to multilayer adsorption. In addition, the presence of hysteresis loop with lower closure point at $p / p 0 \sim 0.4$ and higher closure point at $p / p 0 \sim 0.9$ is related to the filling and emptying of mesopores by capillary condensation. The porous structure characteristics of C-CNC and C-Lignin are listed in Table 1.

There are significant differences between these carbonized samples. The surface area of C-CNC sample calculated from QSDFT and BET methods ranges between 556 and $562 \mathrm{~m}^{2} / \mathrm{g}$. In contrast, C-Lignin has much lower surface area, in the range of $32-47 \mathrm{~m}^{2} / \mathrm{g}$. Furthermore, C-CNC has a larger specific pore volume than C-Lignin does. The pore size distribution of C-CNC shows a small fraction of micropores $(<2 \mathrm{~nm})$ and a large proportion of mesopores $(2-50 \mathrm{~nm})$ 

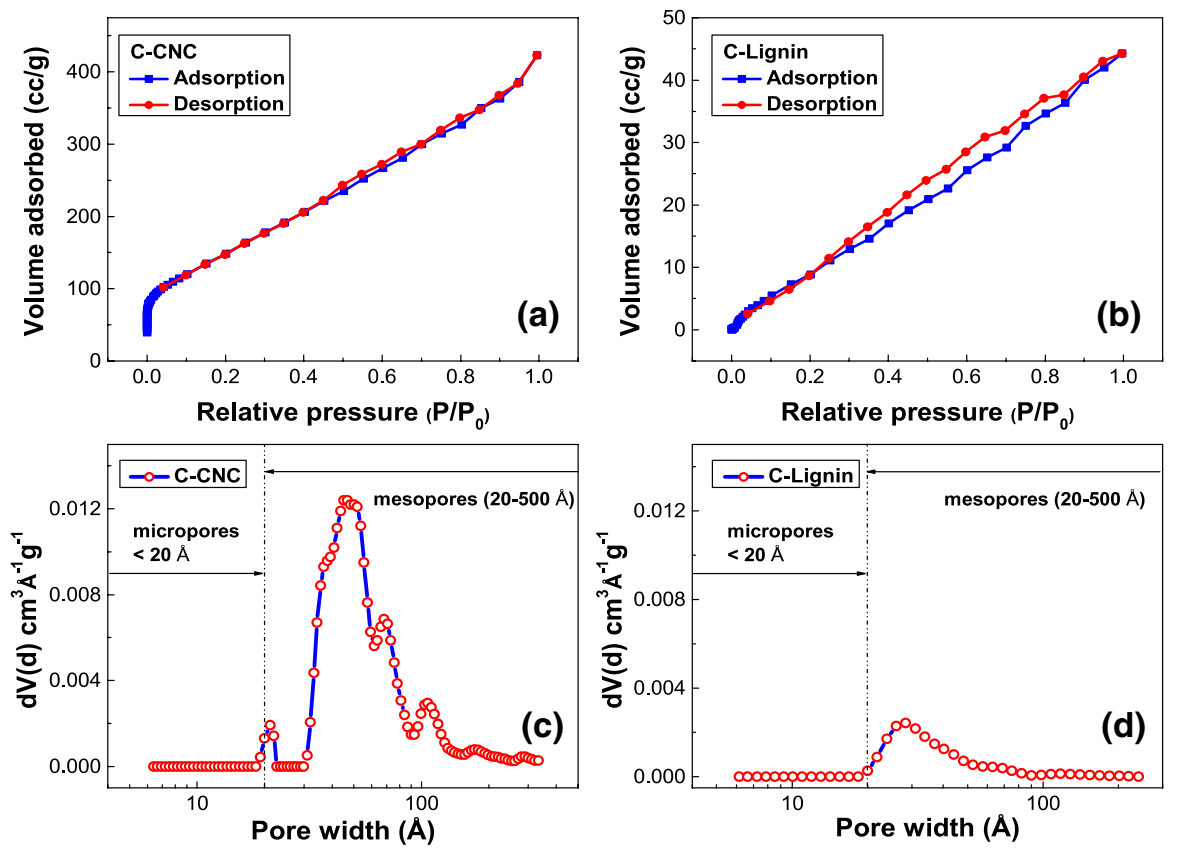

Fig. 1 Adsorption-desorption isotherms of nitrogen on a C-CNC and b C-Lignin: square dotted and round dotted lines are adsorption and desorption data, respectively. Pore size distributions determined by QSDFT method for $\mathbf{c}$ C-CNC and $\mathbf{d}$ C-Lignin

Table 1 Pore surface area and pore volumes of as-prepared C-CNC and C-Lignin

\begin{tabular}{|c|c|c|c|c|}
\hline \multirow{2}{*}{$\begin{array}{l}\text { BET surface area } \\
\left(\mathrm{m}^{2} / \mathrm{g}\right)\end{array}$} & \multicolumn{4}{|c|}{ Results from QSDFT analysis } \\
\hline & $\begin{array}{l}\text { Pore surface area } \\
\left(\mathrm{m}^{2} / \mathrm{g}\right)\end{array}$ & $\begin{array}{l}\text { Pore volume } \\
(\mathrm{cc} / \mathrm{g})\end{array}$ & $\begin{array}{l}\text { Average pore } \\
\text { diameter }(\AA)\end{array}$ & $\begin{array}{l}\text { Volume-to- } \\
\text { area ratio } \\
(\AA)\end{array}$ \\
\hline 556 & 562 & 0.60 & 43.7 & 10.6 \\
\hline 47.7 & 32.2 & 0.06 & 28.4 & 18.6 \\
\hline
\end{tabular}

centered at about $4.4 \mathrm{~nm}$. On the contrary, C-Lignin exhibits a broad pore size distribution, with a smaller volume of mesopores centered at about $2.8 \mathrm{~nm}$. The ratio of total pore volume to BET area is larger for C-Lignin than for C-CNC. This might be the result of the loose packing of C-Lignin particles, associated with interparticle voids rather than intraparticle pores. As such, it can be speculated that the reason for the difference in adsorption properties may be related to the variance of morphological structure between C-CNC and C-Lignin. 


\section{Observation of cellulose nanocrystal and lattice structure}

Gentle STEM was used to study the structure of original CNCs. The TEM image in Fig. 2a shows typical spindle-like whiskers with length in the range of 100-300 nm and width in 3-5 nm. Aggregated bundles of CNC were also observed due to the lateral adhesion among elementary crystallites. The bright-field (BF) higher-magnification image in Fig. 2b clearly shows that CNCs are composed of highly ordered structures. Large domains of cellulose chains were packed tightly together with a high degree of alignment. The formation of cellulose crystalline region can be ascribed to the intra- and intermolecular hydrogen bonds that holds closely packed glucose chains (Nishiyama et al. 2002). The gentle STEM experiment confirms the CNCs' crystalline structure by direct observation. The spacing of lattice fringes is measured to be $\sim 0.4 \mathrm{~nm}$, which is consistent with the $d$-spacing value $(0.39 \mathrm{~nm})$ of the (200) lattice plane of cellulose I.

Observing cellulose nanocrystal without negative staining by STEM is quite challenging due to the nature of the material and possible sample contamination. Figure 3 illustrates the beam irradiation effect on the CNCs. It is of interest to note that these lattice features are not sustained under electron beam, even though the accelerating voltage of the microscope is only $60 \mathrm{kV}$. The fast Fourier transform (FFT) pattern shown in Fig. 3a indicates the good crystallinity of the original CNCs. Shortly after the observation, the crystal structure started to disappear and tended to become amorphous, as seen in Fig. 3b. This is not surprising, since under $60 \mathrm{keV}$ electron beam irradiation, the maximum energy transfer to a carbon atom is about $10.8 \mathrm{eV}$, which is larger than the bonding energy of hydrogen bonds $(<0.5 \mathrm{eV})$ and the activation energy for depolymerization of cellulose (2.68 eV) (Lee et al. 2013; Shen et al. 2013). Accordingly, it is more likely that the high energy of electron beam breaks the intermolecular hydrogen bonds, which bonds the linear chains of $\beta$ $(1,4)$ glucose molecules.
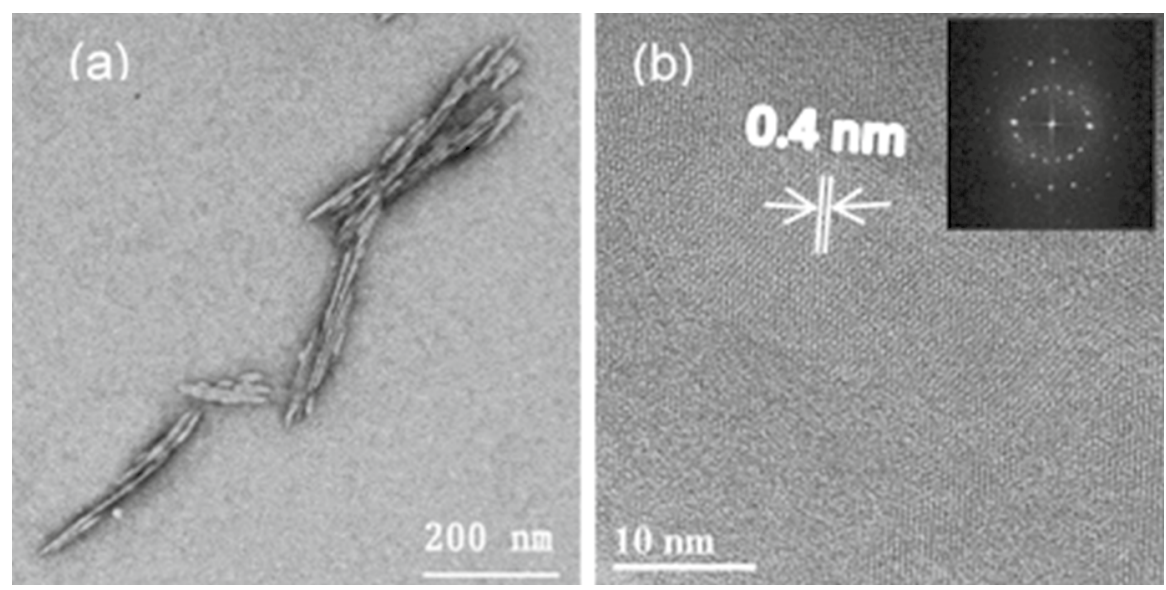

Fig. 2 TEM image of individual CNCs (a) and higher-magnification BF image of its lattice structure (b). The inset in the right upper corner is a fast Fourier transform (FFT) pattern 


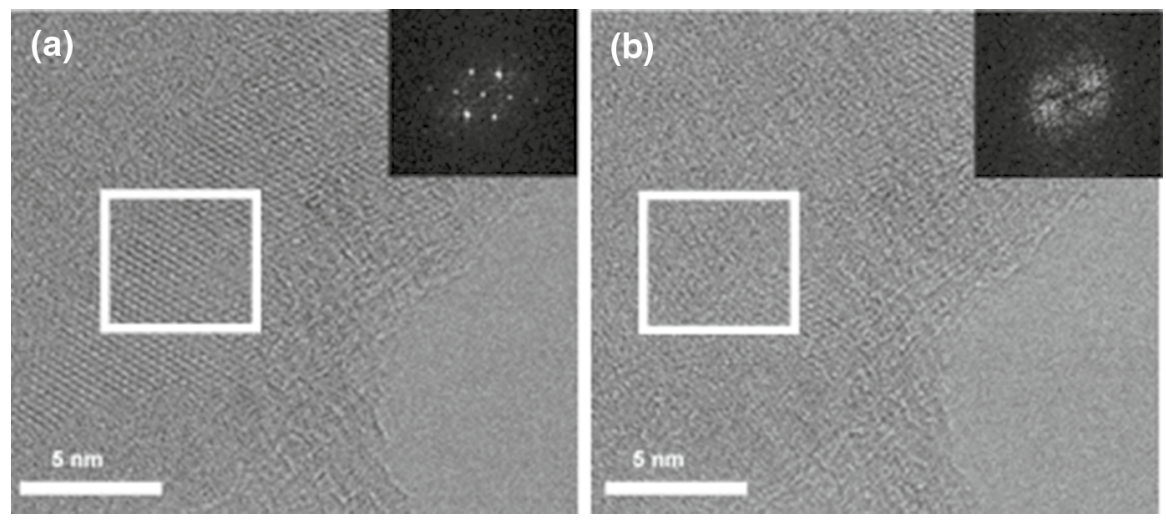

Fig. 3 BF STEM image of CNCs before (a) and after (b) beam irradiation. The inset in the right upper corner is a fast Fourier transform (FFT) pattern

\section{Observation of graphene-like structures in carbonized cellulose nanocrystal and carbonized lignin}

Figure $4 \mathrm{a}, \mathrm{b}$ shows high-resolution STEM images of carbonized C-CNC and $\mathrm{C}$-Lignin. The fringes visible in these images represent an edge-on view of graphene-like layers. An obvious difference between the microstructures of C-CNC and C-Lignin can be seen by comparing Fig. 4a, b. The C-CNC sample consists of short, randomly orientated graphene fringe fragments. The random arrangement of these small fragments creates numerous voids that form the network of interconnected micropores and mesopores. In contrast, a turbostratic carbon structure was not observed in C-Lignin sample. This sample exhibits two types of carbon onion structures (Guo et al. 2009). Figure 4b shows ribbon-like structures composed of 3-4 layers of parallel-stacked carbon layers forming continuous loops, about 15-30 nm long. Similar structures were reported by other researchers (Johnson et al. 1975; Barin et al. 2014). Others proposed that carbonized kraft lignin has the tendency to form nanofringes as temperature increased from 500 to $900{ }^{\circ} \mathrm{C}$; however, no long ribbon-like structures of fringes were observed (Zhang et al. 2017). The discrepancy could be caused from the resources of kraft lignin, where a complicated and large number of process variables are involved when extracting lignin.

In contrast, such large regions of closed packed layers have never been observed in the C-CNC sample. The second component appears with high incidence in numerous images, such as the one shown in Fig. 4c. These faceted onion-like particles are pentagonal or hexagonal in shape. The black circular spot marked by arrow in Fig. 4c is about $10 \mathrm{~nm}$ in diameter; the interplanar distance measured for the dense core is $0.34 \mathrm{~nm}$. The diffraction pattern shown in Fig. 4d confirms the existence of periodic crystal structures in C-Lignin sample.

The microstructural features observed from the electron microscopy observations explain the differences in BET surface area and pore size distribution between $\mathrm{C}-\mathrm{CNC}$ and $\mathrm{C}$-Lignin. These observations indicate that, under identical 

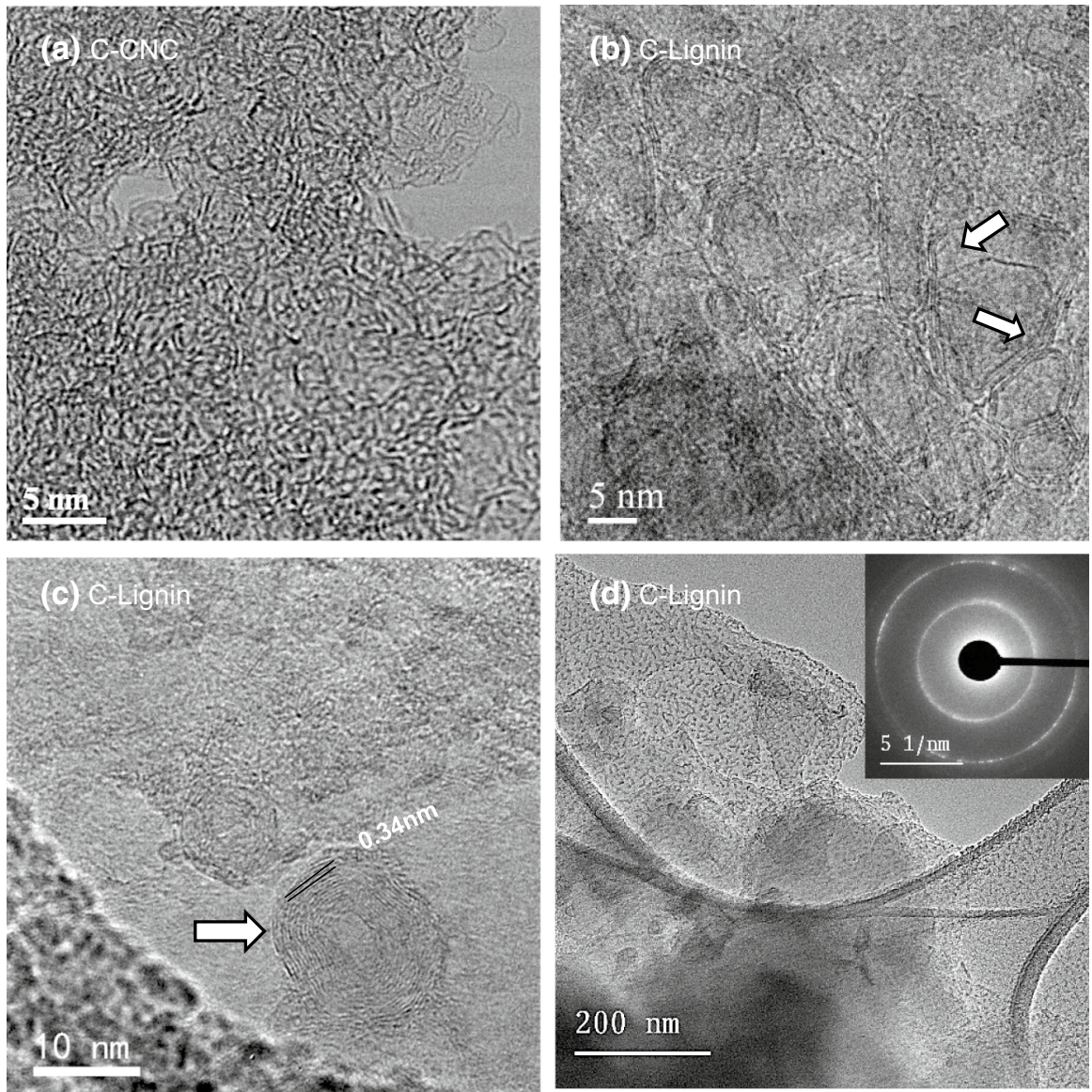

Fig. 4 Micrographs of $\mathrm{CNC}$ carbon and lignin carbon following heat treatment at $950{ }^{\circ} \mathrm{C}$ : Bright-field STEM images of a C-CNC, $\mathbf{b}$ C-Lignin, $\mathbf{c}$ and $\mathbf{d}$ C-Lignin under low magnification, The inset in the right upper corner of $\mathbf{d}$ is the diffraction pattern

carbonization conditions, the transformation of lignin structure into a well-ordered, graphite sheet structure is more facile when compared to that of $\mathrm{CNC}$. That is, the structure and chemical nature of precursor materials (cellulose, lignin) have a pronounced effect on the conversion process and the structure of the carbonized product.

Atomic-resolution annular dark-field (ADF) images were obtained from the very thin regions at the edge of carbonized CNCs particles. Figure 5a shows a low-magnification image of the C-CNC structure. Clearly, the stacking configuration of the graphene-like structure can be described as a pack of numerous, randomly curved atom-thick layers. In order to clearly resolve the carbon atoms in the graphite-like plane in the image, the contribution of the high-frequency noise and electron probe "tails" to the raw ADF images has been removed (using a Gaussianbased probe deconvolution algorithm) (Lee et al. 2013). Careful examination at high 

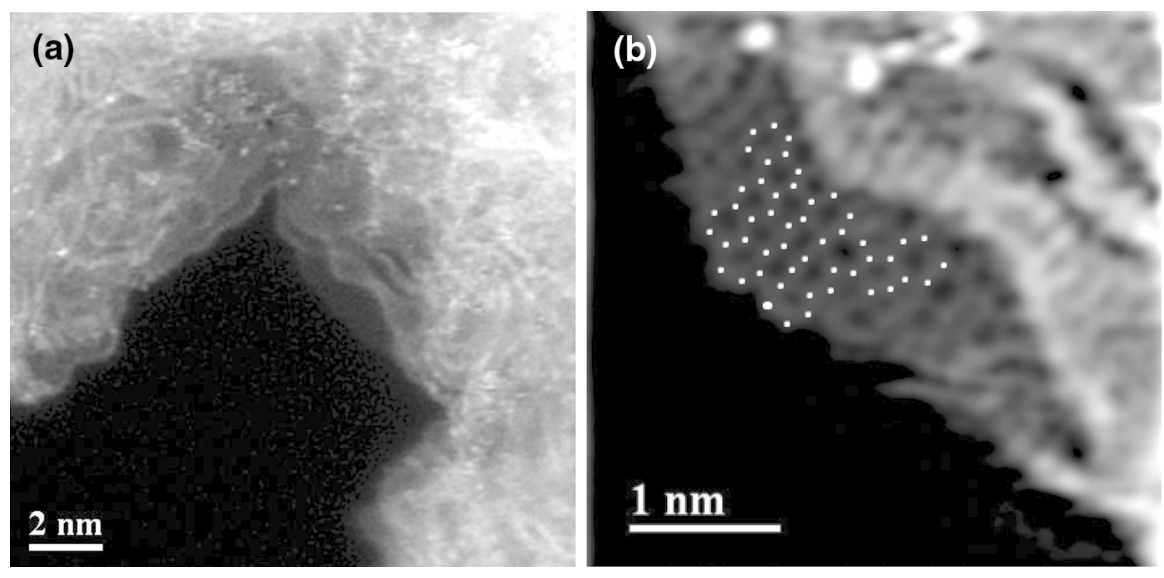

Fig. 5 ADF images obtained from thin edge area of carbonized cellulose nanocrystals: a low magnification (left) and b high magnification (right)

magnification reveals a carbonized $\mathrm{CNC}$ structure. The in-plane carbon atoms are clearly resolved as illustrated in Fig. 5b from a small single-layer graphite/graphene sheet structure suspended on one particle edge and oriented parallel to the image plane. To better analyze the atomic arrangement, each bright spot representing a carbon atom was marked with a white dot on the image. The one-layer graphene/ graphite sheet structure is composed of a hexagonal lattice of carbon atoms, which also contains non-hexagonal defects (atoms are bonded in pentagonal or heptagonal rings). Studies on nanoporous carbon derived from wood-based material revealed domains of imperfectly stacked graphene layers with rippled shapes. The topological defects represented by non-hexagonal carbon atom rings generated highly porous structure with high surface area (Lee et al. 2013). The current observation on C-CNC sample is consistent with the previous report, indicating that as-prepared $\mathrm{C}-\mathrm{CNC}$ is composed of randomly curved graphene layers. Their presence explains the higher BET surface area and porosity of C-CNC.

\section{Thermogravimetric analysis (TG)}

Thermogravimetric analysis (TG) was used for better understanding of carbonization mechanism differences between cellulose and lignin. The temperature profile mimicked the tube furnace carbonization process. In Fig. 6, the weight loss (TG) and derivative of weight loss (DTG) during carbonization are highlighted. It is generally agreed that the process of cellulose pyrolysis consists of four stages: (1) physical desorption of water $\left(25-150{ }^{\circ} \mathrm{C}\right)$; (2) dehydration of the cellulose unit $\left(150-240{ }^{\circ} \mathrm{C}\right)$; (3) thermal cleavage and scission $\left(240-400{ }^{\circ} \mathrm{C}\right)$; and (4) aromatization and carbonization $\left(400{ }^{\circ} \mathrm{C}\right.$ and above) (Meng et al. 2014; Tang and Bacon 1964). These four stages are illustrated in Fig. 6. A major slope change of the TG curve was observed between 180 and $400{ }^{\circ} \mathrm{C}$, consistent with the TGA analysis (Cao et al. 2009) for CNC extracted from cotton by sulfuric acid hydrolysis. Two 

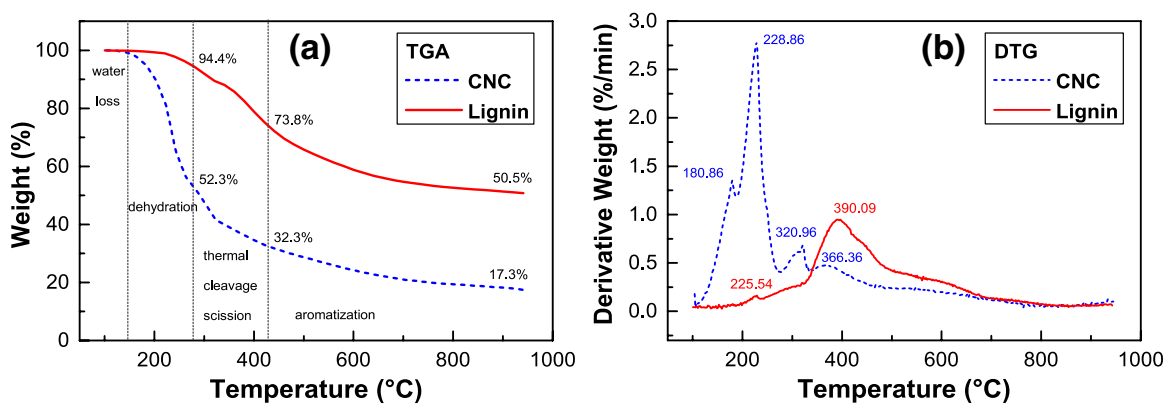

Fig. 6 Typical TG-DTG curves in N2 for cellulose nanocrystals and lignin: a percentage weight loss as a function of temperature and $\mathbf{b}$ weight loss rate as a function of temperature

sharp peaks in the DTG plots at $180.9^{\circ} \mathrm{C}$ and $228.9^{\circ} \mathrm{C}$, accompanied by weight loss of $4.4 \%$ and $21.8 \%$, were attributed to water evaporation and dehydration, respectively (Dumanli and Windle 2012). The DTG peak at $320.9{ }^{\circ} \mathrm{C}$ and the broad peak between 350 and $400{ }^{\circ} \mathrm{C}$ can be traced to complicated reactions of depolymerization and aromatization through the whole carbonization process (Tang and Bacon 1964). In contrast, lignin shows a stepwise decrease of the TG curve in a broad temperature range from about 250 to $700{ }^{\circ} \mathrm{C}$. Unlike the $\mathrm{CNC}$ material, the DTG curve for lignin shows a single broad peak centered at about $390{ }^{\circ} \mathrm{C}$, corresponding to $20 \%$ weight loss. A similar trend was reported by others (Yang et al. 2007). The onset temperatures, decomposition temperatures, and the total weight loss at $950^{\circ} \mathrm{C}$ are summarized in Table 2 .

Compared to lignin, CNC sample started to decompose at lower temperature. The FTIR spectra of the CNC and lignin sample and of their corresponding carbonized materials (Fig. 7) show that the oxygen-containing functional groups were thoroughly removed by thermal treatment at $950^{\circ} \mathrm{C}$.

\section{X-ray diffraction (XRD) analysis}

The XRD pattern of CNC shows typical cellulose peaks marked at $2 \theta=22.5^{\circ}$, $14.6^{\circ}$ and $16.5^{\circ}$, corresponding to its crystal lattice assignment of (200), (1-10) and (110) crystalline planes (Fig. 8). Crystallinity was calculated to be of $81 \%$ using Eq. (1). Carbonization treatment at $950^{\circ} \mathrm{C}$ of $\mathrm{CNC}$ sample leads to the

Table 2 Onset temperature, degradation temperature, maximum weight loss rates and weight loss of $\mathrm{CNC}$ and lignin

\begin{tabular}{lllll}
\hline Carbon precursor & $\begin{array}{l}\text { Onset tempera- } \\
\text { ture }\left({ }^{\circ} \mathrm{C}\right)\end{array}$ & $\begin{array}{l}\text { Degradation tem- } \\
\text { perature }\left({ }^{\circ} \mathrm{C}\right)\end{array}$ & $\begin{array}{l}\text { Maximum weight loss } \\
\text { rate }(\% / \mathrm{min})\end{array}$ & $\begin{array}{l}\text { Weight loss } \\
\text { at } 950{ }^{\circ} \mathrm{C} \\
(\%)\end{array}$ \\
\hline $\mathrm{CNC}$ & 186.0 & 228.9 & -2.82 & 82.6 \\
Lignin & 247.7 & 390.0 & -0.98 & 49.3 \\
\hline
\end{tabular}



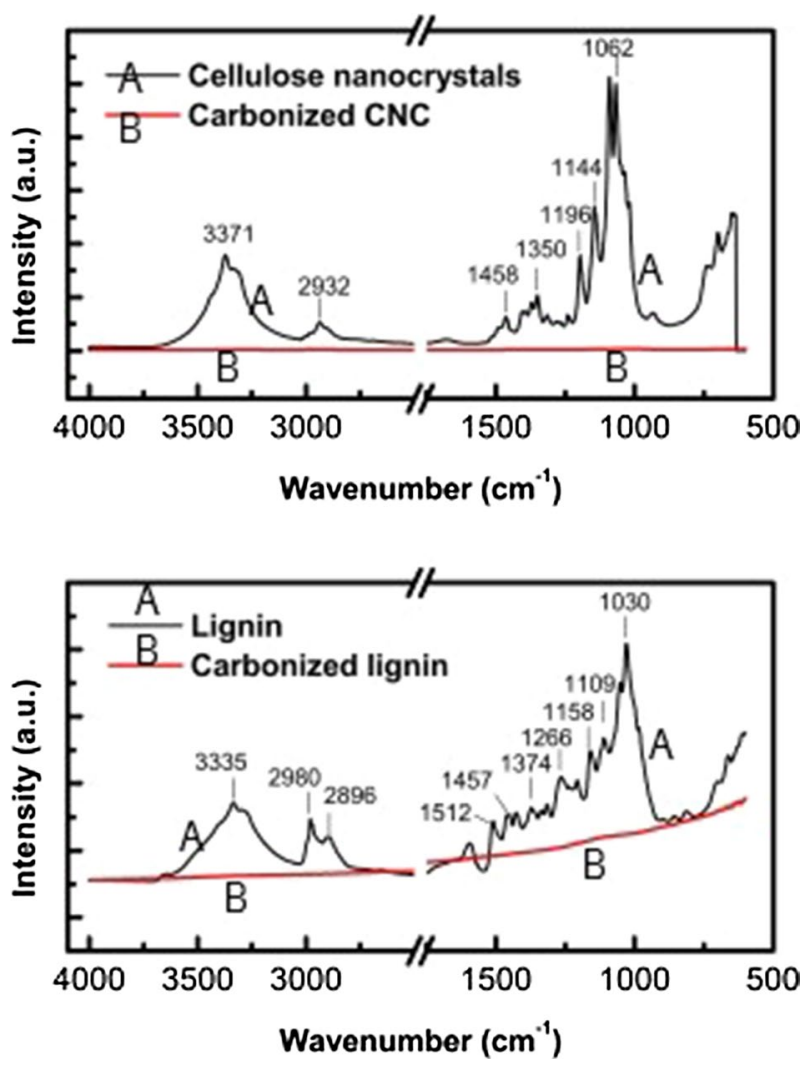

Fig. 7 FTIR spectra of a cellulose nanocrystals and C-CNC (b), lignin and C-Lignin

disappearance of (200), (1-10) and (110) diffraction peaks. A weak, broad peak centered at $2 \theta=17^{\circ}$ was observed, indicating the formation of very disordered, turbostratic-like carbon after carbonization.

Lignin sample's XRD pattern barely exhibits a weak and broad peak centered at $16^{\circ}$, indicating the amorphous nature of lignin. The weak peak shifted to higher $2-\theta$ of $21.2^{\circ}$ upon carbonization treatment, but did not reach $26^{\circ}$, which is the approximate position of (002) peak in graphite. This peak shift accompanied with the decrease in peak width indicates the trend of carbon layers stacking along the $c$-axis (Fujimoto 2003). Similar peak profile was observed in other research (Tenhaeff et al. 2014). Using the Scherrer equation for the (002) peak, an average crystallite length Lc along $c$-axis of $1.1 \mathrm{~nm}$ was calculated. The interlayer spacing (d200) value for C-Lignin was calculated to be $0.37 \mathrm{~nm}$, close to the value $(0.34 \mathrm{~nm})$ measured from the TEM image and the diffraction pattern. The resulted layer, which was calculated as the ratio of Lc and $\mathrm{d} 200$, is 3. It agrees very well with the observation from TEM image in Fig. 4b. However, the incidence of long-range well-ordered graphene stacks remains low, even though they were captured by TEM images. 

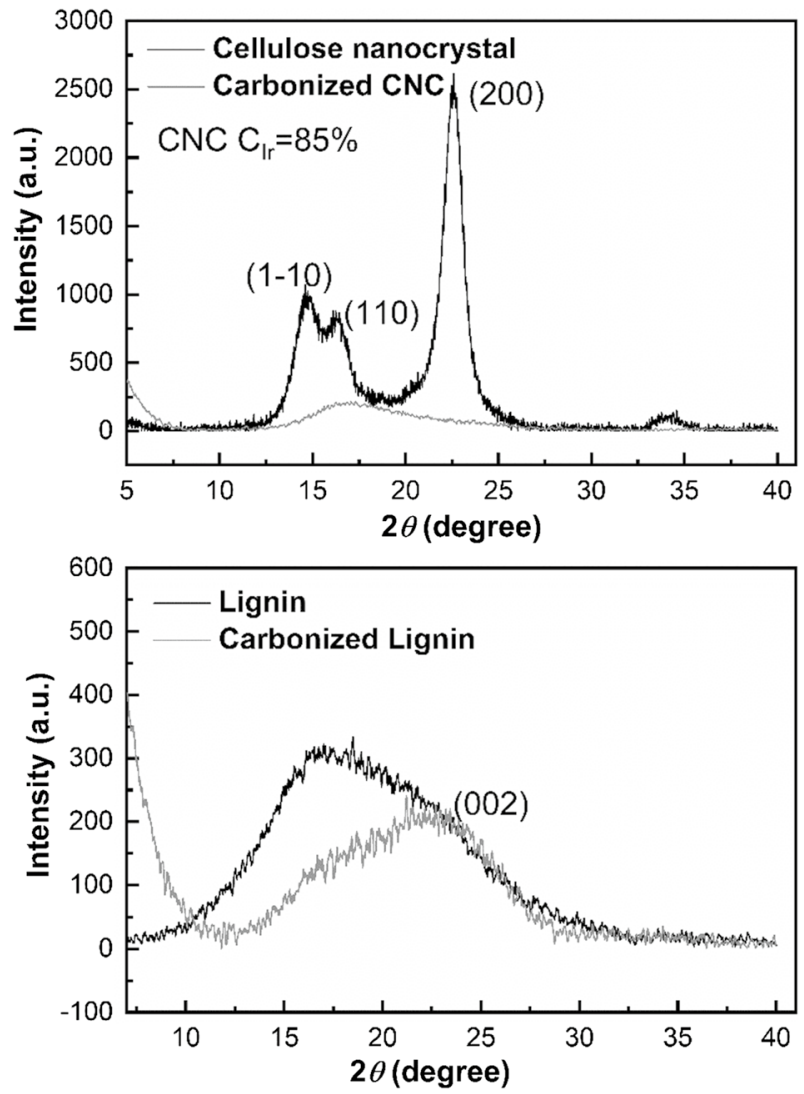

Fig. 8 Wide-angle X-ray diffraction curves of CNC, C-CNC, lignin and C-Lignin

Figure 9 shows the Raman spectra of carbonized CNC and carbonized lignin. The peaks were deconvoluted, and Table 3 summarizes the $I_{D} / I_{G}$ intensity ratio for carbonized CNC. The calculated intensity ratio for carbonized $\mathrm{CNC}$ is 1.52 , which is greater than that of carbonized lignin of $1.23 \mathrm{~A}$. Larger intensity ratio indicates that carbonized CNC sample exhibits a larger quantity of the disordered graphite structure. Likewise, a weak 2D band was observed in the carbonized lignin sample, indicating that there are well-crystallized graphene layers.

Despite lignin's structural and chemical variability, its branched and cross-linked structure consists of only three basic units, derived from coumaryl alcohol, coniferyl alcohol, and sinapyl alcohol (Thielemans et al. 2002). The high level of lignin's aromaticity contributes to its resistance to thermal degradation, as supported by low weight loss on TGA analysis. The HRTEM image of C-Lignin (Fig. 4) suggests lignin's ability for formation of close packed graphene/graphite-stacked structures by carbonization. It can be argued that such preferential formation is related to the presence of aromatic monolignols in lignin. A possible path for lignin carbonization is presented in Fig. 10. The large amount of aromatic ring structures 


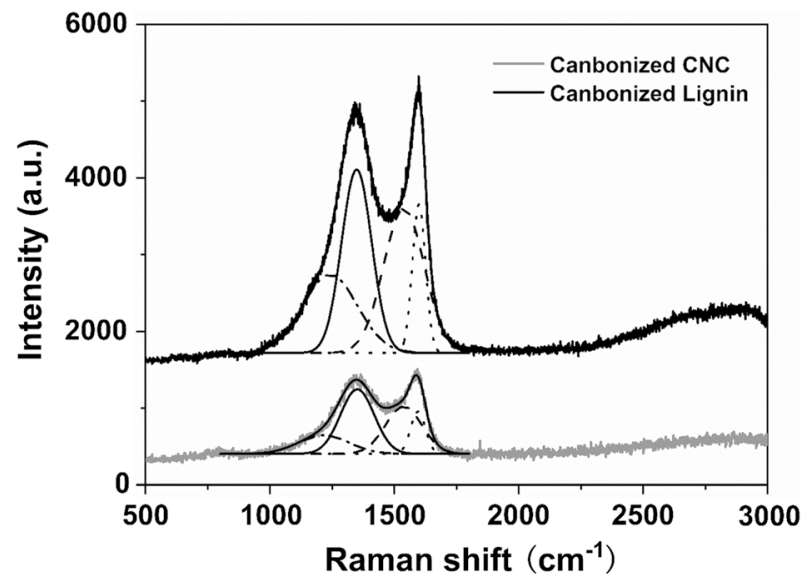

Fig. 9 Raman spectra of carbonized $\mathrm{CNC}$ and carbonized lignin

Table 3 Summary of carbonized CNC and carbonized lignin Raman peak and intensity ratio

\begin{tabular}{|c|c|c|c|c|c|c|c|c|c|}
\hline \multirow[t]{2}{*}{ Sample } & \multicolumn{6}{|l|}{$\mathrm{D}$} & \multicolumn{2}{|l|}{ G } & \multirow[t]{2}{*}{$I_{D} / I_{C}$} \\
\hline & $D_{1}$ & $I_{1}$ & $D_{2}$ & $I_{2}$ & $D_{3}$ & $I_{3}$ & $G$ & $I$ & \\
\hline Carbon & 1216.5 & 243.2 & 1351.1 & 841.9 & 1537.6 & 618.8 & 1595.3 & 533.3 & 1.52 \\
\hline Carbonized Lignin & 1241.9 & 1018.9 & 1348.5 & 2389.3 & 1538.4 & 1879.4 & 1598.9 & 1934.6 & 1.23 \\
\hline
\end{tabular}

$I_{D}$ and $I_{G}$ are intensity values of $D$ and $G$ peaks, respectively.

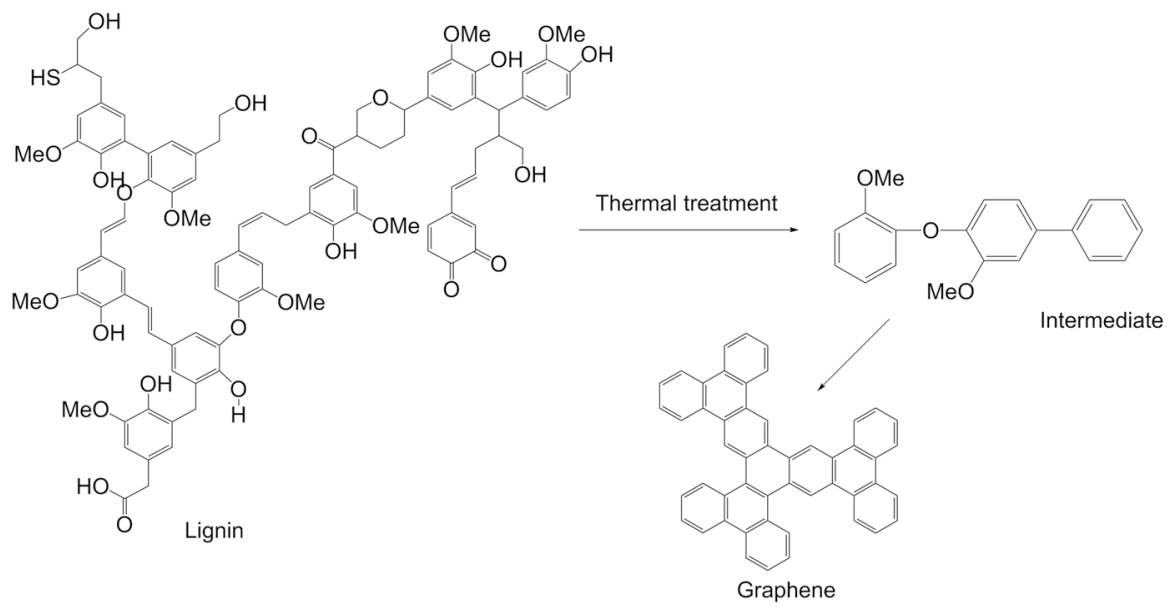

Fig. 10 Schematic of lignin structure (adopted from Ball 1992) evolution during carbonization 
with non-carbon atoms on edges favors aromatic condensation and sequential threedimensional ordering during carbonization and heat treatment up to $950{ }^{\circ} \mathrm{C}$.

The structure of high crystallinity cellulose (CNC), by contrast, is composed of repeating glucose units. A scheme of structural changes during cellulose carbonization is presented in Fig. 11. During heat treatment from room temperature to $950{ }^{\circ} \mathrm{C}$, cellulose experiences desorption of physisorbed water, intermolecular dehydration through $-\mathrm{H}$ and $-\mathrm{OH}$ condensation, thermal cleavage of glycosidic linkages, thermal scission of ether bonds and $\mathrm{C}-\mathrm{C}$ bonds, etc. Davidson and Losty (1963) argued that oxygen existing in pyranose rings and glycoside links is eliminated during the decomposition process, making the main contribution to the total weight loss (Davidson and Losty 1963). Large amounts of carbonaceous intermediates consisting of aromatic platelets terminated by hydrogen would go through further thermal

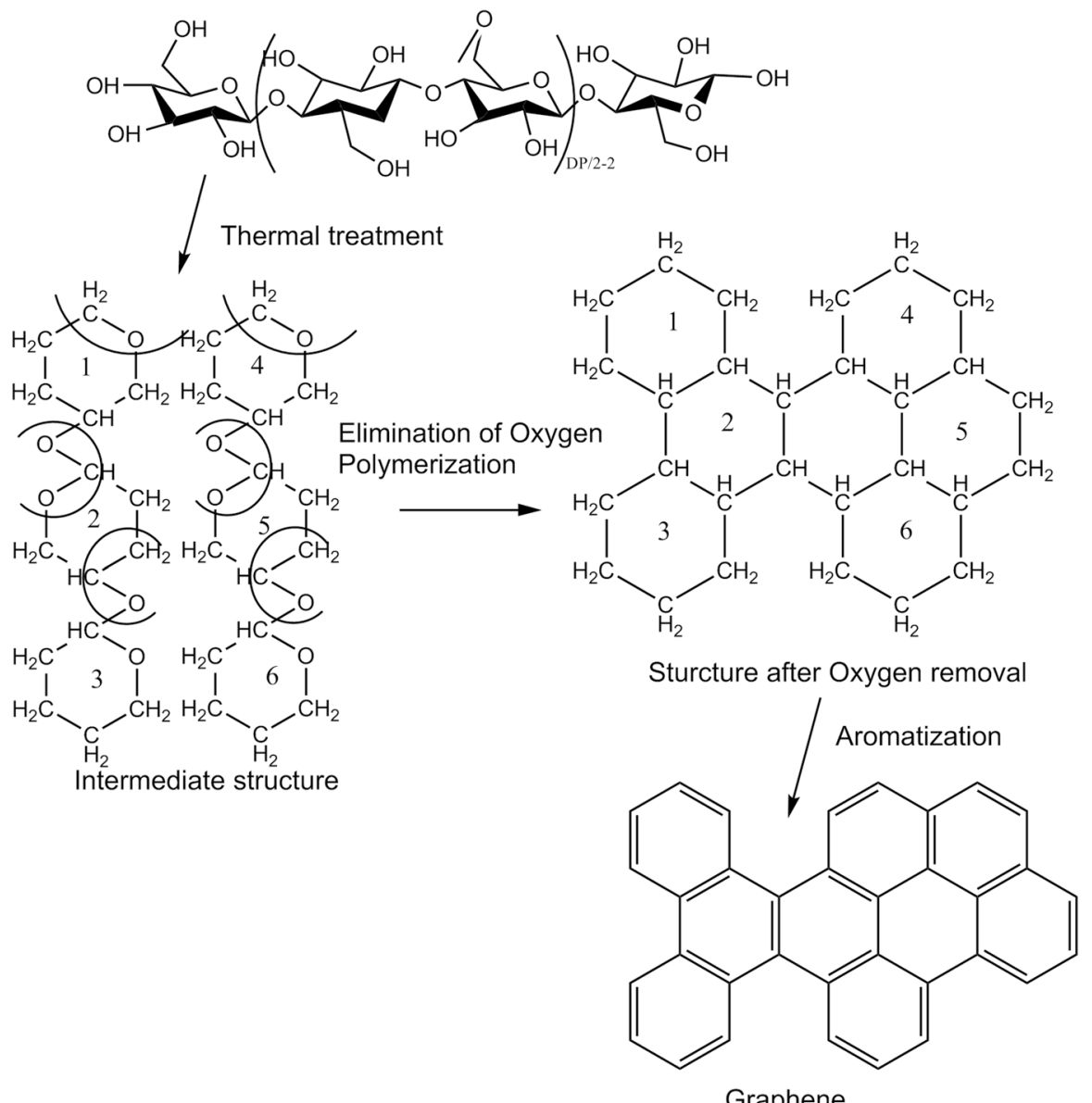

Fig. 11 Scheme of cellulose structure and cellulose carbonization (adopted from Davidson and Losty 1963) 
cleavage above $300{ }^{\circ} \mathrm{C}$. Based on the TEM image (Fig. 4), it can be speculated that cellulose intermediates polymerize, to successfully form graphene/graphite units, yet fail to yield long-range stacking order. The oxygen elimination process requires high reactive energy to break the rings. In addition, the presence of covalently bonded hydrogen may hamper the aromatic condensation.

\section{Conclusion}

This research investigated the structure of carbonized cellulose and carbonized lignin at atomic scale thermally treated at a maximum temperature of $950{ }^{\circ} \mathrm{C}$ by scanning transmission electron microscopy. There is evidence from the study that carbon derived from highly crystallized cellulose yields disordered porous structure, while kraft lignin derived carbon has the tendency to yield larger graphite sheet and onion-like structures. The presence of certain well-ordered carbon in carbonized lignin indicates some of the carbon in lignin could be graphitized with heat treatment temperature. This result is encouraging for the future prospects of attaining acceptable modulus of carbon fiber from lignin given correct modifications to the chemistry and structure of lignin. The results of the study contribute to an improved understanding of the carbonization mechanism of key cellulose and lignin components of biomass materials.

Acknowledgements The authors gratefully acknowledge the support of this research by UTIA 2014 Innovation Grant, USDA Special Wood Utilization Grants R11-0515-041 and R11-2219-510, the University of Tennessee, Department of Forestry, Wildlife and Fisheries; Center for Renewable Carbon, and the Agricultural Experiment Station McIntire-Stennis Grant TENOOMS-101. CIC acknowledges support from the US Department of Energy, Basic Energy Sciences, Materials Science and Technology Division for materials characterization, data analysis, and interpretation. This research used facilities provided by ORNL's Center for Nanophase Materials Sciences (CNMS), which is sponsored by the Scientific User Facilities Division, Office of Basic Energy Sciences, U.S. Department of Energy.

\section{Declarations}

Conflict of interest On behalf of all authors, the corresponding author states that there is no conflict of interest.

Open Access This article is licensed under a Creative Commons Attribution 4.0 International License, which permits use, sharing, adaptation, distribution and reproduction in any medium or format, as long as you give appropriate credit to the original author(s) and the source, provide a link to the Creative Commons licence, and indicate if changes were made. The images or other third party material in this article are included in the article's Creative Commons licence, unless indicated otherwise in a credit line to the material. If material is not included in the article's Creative Commons licence and your intended use is not permitted by statutory regulation or exceeds the permitted use, you will need to obtain permission directly from the copyright holder. To view a copy of this licence, visit http://creativecommons.org/licen ses/by/4.0/. 


\section{References}

Aarum I, Solli A, Gunnarsson H, Kalyani D, Devle H, Ekeberg D, Stenstrøm Y (2019) Comparison of pyrolyzed lignin before and after milled wood lignin purification of Norway spruce with increasing steam explosion. Wood Sci Technol 53(3):601-618. https://doi.org/10.1007/s00226-019-01088-X

Aina V, Alberto G, Deiana C, Sakhno Y, Damin A, Martra G (2013) Surface sites of nanomaterials: investigation of local structures by In situ IR spectroscopy. In: Fesenko O, Yatsenko L, Brodin M (eds) Nanomaterials imaging techniques, surface studies, and applications, vol 146. Springer, Berlin, pp 145-163

Ball FJ (1992) Chemistry of lignin and its applications, Technical Literature. Westvaco Chemical Division: Charleston Heights

Barin GB, Gimenez IF, Costa LP, Filho AGS, Barreto LS (2014) Influence of hydrothermal carbonization on formation of curved graphite structures obtained from a lignocellulosic precursor. Carbon 78:609-612. https://doi.org/10.1016/j.carbon.2014.07.017

Cao X, Habibi Y, Lucia LA (2009) One-pot polymerization, surface grafting, and processing of waterborne polyurethane-cellulose nanocrystal nanocomposites. Chemistry 19(38):7137-7145. https://doi.org/10.1039/B910517D

Cao J, Xiao G, Xu X, Shen D, Jin B (2013) Study on carbonization of lignin by TG-FTIR and hightemperature carbonization reactor. Fuel Process Technol 106:41-47. https://doi.org/10.1016/j. fuproc.2012.06.016

Chen C, Sun K, Wang A, Sun H, Lu X, Xu W, Wang S, Jiang J (2019) Preparation of photocatalyst with high reactivity from cellulose-based graphitic carbon. BioResources 14(3):7213-7228

Childres I, Jauregui LA, Park W, Cao H, Chen YP (2013) Raman spectroscopy of graphene and related materials. In: Jang JI (ed) New developments in photon and materials research. Nova Science Publishers, Hauppauge, ISBN:978-1-62618-339-1

Davidson H, Losty H (1963) The initial pyrolyses of cellulose. GEC J 30:22-28

Dobele G, Dizhbite T, Gil MV, Volperts A, Centeno TA (2012) Production of nanoporous carbons from wood processing wastes and their use in supercapacitors and $\mathrm{CO}_{2}$ capture. Biomass Bioenergy 46(2012):145-154. https://doi.org/10.1016/j.biombioe.2012.09.010

Dumanli AJ, Windle AH (2012) Conductive carbon microfibers derived from wet-spun lignin/nanocellulose hydrogels. J Mater Sci 47(10):4236-4250. https://doi.org/10.1007/s10853-011-6081-8

Fujimoto H (2003) Activated carbons for applications in catalysis: the point of view of a physicalchemist. Carbon 41(8):1585-1592. https://doi.org/10.1016/S0008-6223(03)00116-7

Goodell B, Xie XF, Qian YH, Daniel G, Peterson M, Jellison J (2008) Carbon nanotubes produced from natural cellulosic materials. J Nanosci Nanotechnol 8(5):2472-2474. https://doi.org/10. 1166/jnn.2008.235

Guo J, Wang X, Xu B (2009) One-step synthesis of carbon-onion-supported platinum nanoparticles by arc discharge in an aqueous solution. Mater Chem Phys 113(1):179-182. https://doi.org/10. 1016/j.matchemphys.2008.07.039

Guo J, Morris JR, Ihm Y, Contescu CI, Gallego NC, Duscher G, Pennycook SJ, Chisholm MF (2012) Topological defects: origin of nanopores and enhanced adsorption performance in nanoporous carbon. Small 8(21):3283-3288. https://doi.org/10.1002/smll.201200894

Hata T, Imamura Y, Kobayashi E, Yamane K, Kikuchi K (2000) Onion-like graphitic particles observed in wood charcoal. J Wood Sci 46(1):89-92. https://doi.org/10.1007/BF00779560

He L, Melnichenko YB, Gallego NC, Contescu CI, Guo J, Bahadur J (2014) Investigation of morphology and hydrogen adsorption capacity of disordered carbons. Carbon 80:82-90. https://doi.org/ 10.1016/j.carbon.2014.08.041

Ishimaru K, Hata T, Bronsveld P, Meier D, Imamura Y (2007) Spectroscopic analysis of carbonization behavior of wood, cellulose and lignin. J Mater Sci 42(1):122-129. https://doi.org/10.1007/ s10853-006-1042-3

Johnson DJ, Tomizuka I, Watanabe O (1975) The fine structure of pitch-based carbon fibres. Carbon 13(6):529-534. https://doi.org/10.1016/0008-6223(75)90056-1

Langan P, Petridis L, O’Neill HM, Pingali SV, Foston M, Nishiyama Y, Schulz R, Lindner B et al (2014) Common processes drive the thermochemical pretreatment of lignocellulosic biomass. Green Chem 16(1):63-68

Lee J, Zhou W, Pennycook SJ, Idrobo J-C, Pantelides ST (2013) Direct visualization of reversible dynamics in a Si6 cluster embedded in a graphene pore. Nat Commun 4:1650 
Liu D, Chen X, Yue Y, Chen M, Wu Q (2011) Structure and rheology of nanocrystalline cellulose. Carbohydr Polym 84(1):316-322. https://doi.org/10.1016/j.carbpol.2010.11.039

Ma L, Zhang Y, Wang S (2016) Modified treatment for carbonized cellulose nanofiber application in composites. Compos A Appl Sci Manuf 90:786-793. https://doi.org/10.1016/j.compositesa. 2016.09.007

Mao S, Lu G, Chen J (2015) Three-dimensional graphene-based composites for energy applications. Nanoscale 7:6924

Meng Y, Young T, Liu P, Contescu C, Huang B, Wang S (2014) Ultralight carbon aerogel from nanocellulose as highly selective oil absorption materials. Cellulose 22(1):435-447. https://doi.org/ 10.1007/s 10570-014-0519-5

Moon R, Martini A, Nairn J, Simonsen J, Youngblood J (2011) Cellulose nanomaterials review: structure, properties and nanocomposites. Chem Soc Rev 40(7):3941-3994. https://doi.org/10.1039/ c0cs00108b

Muramatsu H, Kim YA, Yang K-S, Cruz-Silva R, Toda I, Yamada T, Terrones M, Endo M, Hayashi T, Saitoh H (2014) Rice husk-derived graphene with nano-sized domains and clean edges. Small 10(14):2766-2770. https://doi.org/10.1002/smll.201400017

Nishiyama Y, Langan P, Chanzy H (2002) Crystal structure and hydrogen-bonding system in cellulose $\mathrm{I} \beta$ from synchrotron X-ray and neutron fiber diffraction. J Am Chem Soc 124(31):9074-9082. https://doi.org/10.1021/ja0257319

Patterson A (1939) The Scherrer formula for x-ray particle size determination. Phys Rev 56(10):978982. https://doi.org/10.1103/PhysRev.56.978

Ragauskas AJ, Williams CK, Davison BH, Britovsek G, Cairney J, Eckert CA, Frederick WJ et al (2006) The path forward for biofuels and biomaterials. Science 311(5760):484-489. https://doi. org/10.1126/science. 1114736

Risnasari I, Febrianto F, Wistara NJ et al (2018) Characterization of cellulose nanocrystal with cellulose II polymorph from primary sludge and its application to PVA nanocomposites. Wood Sci Technol 52:555-565. https://doi.org/10.1007/s00226-017-0976-8

Shen D, Xiao R, Zhang H, Gu S (2013) The overview of thermal decomposition of cellulose in lignocellulosic biomass. In: van de Ven J, Kadla J (eds) Cellulose-biomass conversion. InTech Open, London. https://doi.org/10.5772/51883

Sing KSW, Everett DH, Haul RAW, Moscou L, Pierotti RA, Rouquerol J, Siemieniewska T (1985) Reporting physisorption data for gas/solid systems with special reference to the determination of surface area and porosity. Pure Appl Chem 57(4):603-619. https://doi.org/10.1351/pac1985570 40603

Tang MM, Bacon R (1964) Carbonization of cellulose fibers-I. Low temperature pyrolysis. Carbon 2(3):211-214. https://doi.org/10.1016/0008-6223(64)90035-1

Tenhaeff WE, Rios O, More K, McGuire MA (2014) Highly robust lithium ion battery anodes from lignin: an abundant, renewable, and low-cost material. Adv Funct Mater 24(1):86-94. https://doi. org/10.1002/adfm.201301420

Thielemans W, Can E, Morye SS, Wool RP (2002) Novel applications of lignin in composite materials. J Appl Polym Sci 83(2):323-331. https://doi.org/10.1002/app.2247

Trache D, Hussin MH, Haafiz MKM, Thakur VK (2017) Recent progress in cellulose nanocrystals: sources and production. Nanoscale 9:1763-1786

Wang H, Xu Z, Kohandehghan A, Li Z, Cui K, Tan X, Stephenson T, King'ondu C, Holt C et al (2013) Interconnected carbon nanosheets derived from hemp for ultrafast supercapacitors with high energy. ACS Nano 7(6):5131-5141. https://doi.org/10.1021/nn400731g

Wang X, Chen H, Feng X, Zhang Q, Kim A, Labbé N, Huang J, Wang S, Zhang Y (2020) Extraction and characterization of lignin-containing cellulose nanofibers from four organosolv-fractionated biomass materials. Wood Sci Technol 54:503-517. https://doi.org/10.1007/s00226-020-01167-4

Wei H, Liao K, Shi P, Fan J, Xu Q (2018) Simple method to construct three-dimensional porous carbon for electrochemical energy storage. Nanoscale 10(33):15842-15853. https://doi.org/10.1039/ c8nr04467h

Wu Q, Meng Y, Concha K, Wang S, Li Y, Ma LF, Fu S (2013) Influence of temperature and humidity on nano-mechanical properties of cellulose nanocrystal films made from switchgrass and cotton. Ind Crop Prod 48:28-35. https://doi.org/10.1016/j.indcrop.2013.03.032

Xiao X, Chen B (2017) A direct observation of the fine aromatic clusters and molecular structures of biochars. Environ Sci Technol 51:5473-5482. https://doi.org/10.1021/acs.est.6b06300 
Xie X, Goodell B, Zhang D, Nagle DC, Qian Y, Peterson ML, Jellison J (2009) Characterization of carbons derived from cellulose and lignin and their oxidative behavior. Bioresource Technol 100(5):1797-1802. https://doi.org/10.1016/j.biortech.2008.09.057

Xu X, Liu F, Jiang L, Zhu JY, Haagenson D, Wiesenborn DP (2013) Cellulose nanocrystals vs. cellulose nanofibrils: a comparative study on their microstructures and effects as polymer reinforcing agents. ACS Appl Mater Int 5(8):2999-3009. https://doi.org/10.1021/am302624t

Yang H, Yan R, Chen H, Lee DH, Zheng C (2007) Characteristics of hemicellulose, cellulose and lignin pyrolysis. Fuel 86(12-13):1781-1788. https://doi.org/10.1016/j.fuel.2006.12.013

Zhang X, Yan Q, Hassan EB, Li J, Cai Z, Zhang J (2017) Temperature effects on formation of carbonbased nanomaterials from kraft lignin. Mater Lett 203:42-45

Zhao P, Wang L, Sun C, Jiang C, Zhang T, Zhang Q, Sun J, Deng Y, Wang S (2012) Uniform mesoporous carbon as a carrier for poorly water soluble drug and its cytotoxicity study. Eur J Pharm Biopharm 80(3):535-543. https://doi.org/10.1016/j.ejpb.2011.12.002

Publisher's Note Springer Nature remains neutral with regard to jurisdictional claims in published maps and institutional affiliations.

\section{Authors and Affiliations}

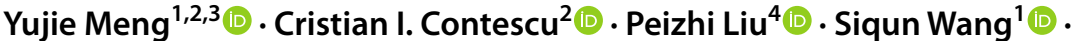 Seung-Hwan Lee ${ }^{5}$. Junjie Guo ${ }^{2,4}$. Timothy M. Young ${ }^{1}$}

Yujie Meng

Yujie.Meng@kla.com

Cristian I. Contescu

contescuci@ornl.gov

Peizhi Liu

liupeizhi@tyut.edu.cn

Siqun Wang

swang@utk.edu

Seung-Hwan Lee

1shyhk@kangwon.ac.kr

Junjie Guo

guojunjie@tyut.edu.cn

1 Center for Renewable Carbon, The University of Tennessee, 2506 Jacob Drive, Knoxville, TN 37996, USA

2 Oak Ridge National Laboratory, Oak Ridge, TN 37831, USA

3 KLA Corporation, Knoxville, TN 37830, USA

4 Key Laboratory of Interface Science and Engineering in Advanced Materials of Ministry of Education, Taiyuan University of Technology, Taiyuan 030024, China

5 Kangwon National University, Chuncheon 200-701, Korea 